TITLE:

\title{
Primitive chain network simulations for asymmetric star polymers
}

$\operatorname{AUTHOR}(S)$ :

Masubuchi, Yuichi; Yaoita, Takatoshi; Matsumiya, Yumi; Watanabe, Hiroshi

\section{CITATION:}

Masubuchi, Yuichi ...[et al]. Primitive chain network simulations for asymmetric star polymers. JOURNAL OF CHEMICAL PHYSICS 2011, 134(19): 194905.

\section{ISSUE DATE:}

2011-05

URL:

http://hdl.handle.net/2433/160658

\section{RIGHT:}

Copyright 2011 American Institute of Physics. This article may be downloaded for personal use only. Any other use requires prior permission of the author and the American Institute of Physics. The following article appeared in JOURNAL OF CHEMICAL PHYSICS 134, 194905 (2011) and may be found at http://link.aip.org/link/?jcp/134/194905 


\section{AIP $\substack{\text { medoumal } \\ \text { chemical Physics }}^{2}$}

\section{Primitive chain network simulations for asymmetric star polymers}

Yuichi Masubuchi, Takatoshi Yaoita, Yumi Matsumiya, and Hiroshi Watanabe

Citation: J. Chem. Phys. 134, 194905 (2011); doi: 10.1063/1.3590276

View online: http://dx.doi.org/10.1063/1.3590276

View Table of Contents: http://jcp.aip.org/resource/1/JCPSA6/v134/i19

Published by the American Institute of Physics.

\section{Additional information on J. Chem. Phys.}

Journal Homepage: http://jcp.aip.org/

Journal Information: http://jcp.aip.org/about/about_the_journal

Top downloads: http://jcp.aip.org/features/most_downloaded

Information for Authors: http://jcp.aip.org/authors

\section{ADVERTISEMENT}

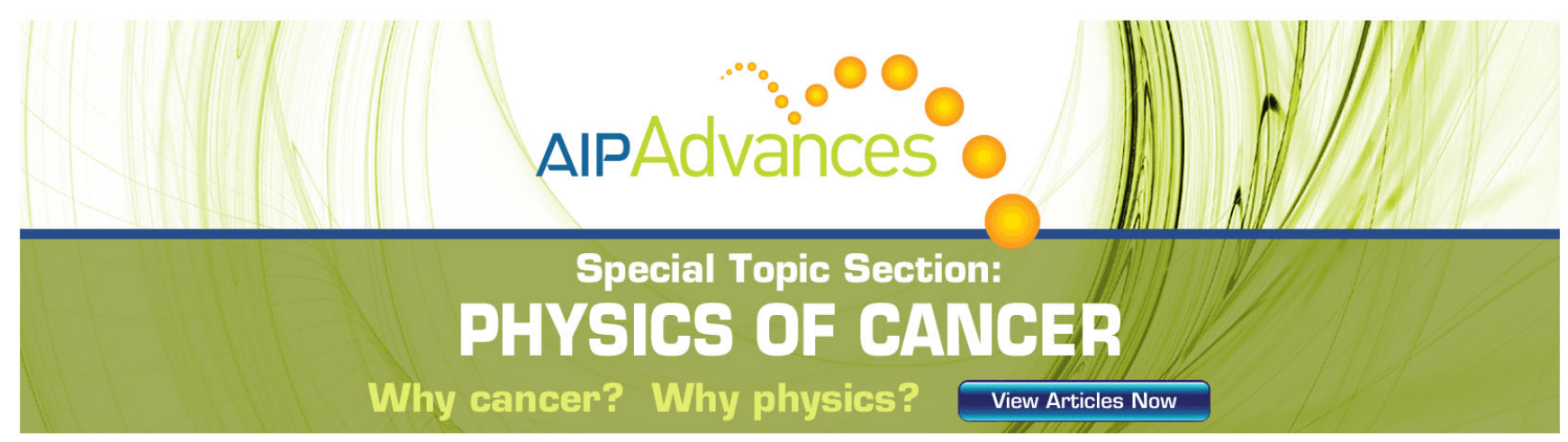




\title{
Primitive chain network simulations for asymmetric star polymers
}

\author{
Yuichi Masubuchi, ${ }^{1,2, a)}$ Takatoshi Yaoita, ${ }^{1,3}$ Yumi Matsumiya, ${ }^{1}$ and Hiroshi Watanabe ${ }^{1}$ \\ ${ }^{1}$ Institute for Chemical Research, Kyoto University, Gokasyo, Uji, Kyoto 611-0011, Japan \\ ${ }^{2}$ JST-CREST, Kyoto 611-0011, Japan \\ ${ }^{3}$ Material Science Laboratory, Mitsui Chemicals Inc., Chiba 299-0265, Japan
}

(Received 22 March 2011; accepted 21 April 2011; published online 17 May 2011)

\begin{abstract}
For branched polymers, the curvilinear motion of the branch point along the backbone is a significant relaxation source but details of this motion have not been well understood. This study conducts multichain sliplink simulations to examine effects of the spatial fluctuation and curvilinear hopping of the branch point on the viscoelastic relaxation. The simulation is based on the primitive chain network model that allows the spatial fluctuations of sliplink and branch point and the chain sliding along the backbone according to the subchain tension, chemical potential gradients, drag force against medium, and random force. The sliplinks are created and/or disrupted through the motion of chain ends. The curvilinear hopping of the branch point along the backbone is allowed to occur when all sliplinks on a branched arm are lost. The simulations considering the fluctuation and the hopping of the branch point described well the viscoelastic data for symmetric and asymmetric star polymers with a parameter set common to the linear polymer. On the other hand, the simulations without the branch point motion predicted unreasonably slow relaxation for asymmetric star polymers. For asymmetric star polymers, further tests with and without the branch point hopping revealed that the hopping is much less important compared to the branch point fluctuation when the lengths of the short and long backbone arms are not very different and the waiting time for the branch point hopping (time for removal of all sliplinks on the short arm) is larger than the backbone relaxation time. Although this waiting time changes with the hopping condition, the above results suggest a significance of the branch point fluctuation in the actual relaxation of branch polymers. (C) 2011 American Institute of Physics. [doi:10.1063/1.3590276]
\end{abstract}

\section{INTRODUCTION}

Attempts have been made for molecular modeling of relaxation of branched polymers. The pioneering study of Doi and $\mathrm{Kuzuu}^{1,2}$ considered the relaxation achieved by the full retraction of the branched arm toward the branch point. This molecular picture is consistent with the experimentally observed exponential dependence of the relaxation time on the arm molecular weight. ${ }^{3}$ The arm retraction model has been refined by Pearson and Helfand ${ }^{4}$ through formulation of the first passage process in the free energy barrier for the arm retraction. The model has been further refined by Ball and McLeish $^{5}$ who analyzed the dynamic tube dilation (DTD) process (coarse-graining process of the effective entanglement segment with increasing time scale) and by Milner and McLeish $^{6}$ through formulation of the first passage process combined with the DTD and incorporation of the fast Rouse relaxation. None of these theories considered the branch point motion explicitly, although the DTD picture automatically allows the branch point to fully fluctuate within a dilated tube diameter.

Since the arm retraction models are not straightforwardly applicable to multi-branch polymers, the hierarchical tube model has been proposed. ${ }^{7}$ This model assumes that the branched arm relaxes first and then the backbone drags the

\footnotetext{
a) Author to whom correspondence should be addressed. Electronic mail: mas@scl.kyoto-u.ac.jp, Tel .:+81-774-38-3136, FAX: +81-774-38-3139.
}

relaxed arms to fully relax through reptation. In this reptation process, the relaxed arm is replaced by a bead being located at the branch point and having the friction proportional to the arm retraction time. Following the remarkable success of the original hierarchical model, ${ }^{7}$ the model has been refined/modified to describe the viscoelastic behavior of star, comb, and asymmetric star polymers. ${ }^{8-13}$

In the hierarchical model, the branch point motion plays a central role but its detail is still controversial. In the early model, ${ }^{7}$ the curvilinear diffusion coefficient of the branch point is given by $D_{\mathrm{b}}=\left(p a_{\mathrm{eff}}\right)^{2} /\left(2 q \tau_{\mathrm{a}}\right)$, where $a_{\mathrm{eff}}$ is the tube diameter dilated through the arm relaxation (retraction), $q$ the number of arms diverging from the branch point, $\tau_{\mathrm{a}}$ the arm relaxation time, and $p$ the parameter specifying the hopping distance, $p a_{\text {eff. }}$. The value of $p^{2}$ was originally considered to be close to unity ${ }^{7}$ but the best fit to the experimental data is achieved with $p^{2}$ significantly smaller than unity. In fact, MD simulation ${ }^{14}$ and neutron spin echo experiment ${ }^{15}$ revealed a very small mobility of the branch point. Nevertheless, the $p^{2}$ value obtained by fitting of the viscoelastic data with the hierarchical model seems to be inconsistent with the hopping picture combined with the full-DTD picture regarding the relaxed portion as a solvent. Specifically, for asymmetric star polymers, $p^{2}$ was found to vary from $1 / 60$ to $1 / 4$ with an increase of the branched arm molecular weight. ${ }^{9}$ This counterintuitive trend of $p^{2}$ has been qualitatively explained by Lee et al. ${ }^{11}$ who compared the relaxation behavior of highly asymmetric and almost symmetric star polymers in the dilated 
tube to propose a relationship, $p^{2}=1 /\left(2 Z_{\mathrm{AR}}\right)$ with $Z_{\mathrm{AR}}$ being the number of unrelaxed entanglement segments of backbone arms at the time $\tau_{\mathrm{a}}$.

Shanbhag and Larson ${ }^{10}$ have proposed a sliplink model not relying on the DTD picture but assuming the branch point to achieve the curvilinear hopping over the undilated tube diameter $a$ when all sliplinks on the short arm are removed. Their model, requiring no fitting parameter for the branch point motion, described well the viscoelastic data for the asymmetric (and symmetric) star polymers. However, the model assumption does not necessarily match the general DTD picture that the branch point unequivocally explores all positions within the distance $>a$ as long as some entanglements are released through relaxation of the arms. This exploration (or fluctuation) could contribute to the relaxation of the portion of the backbone arms near the branch point.

For asymmetric star polymers, the curvilinear hopping of branch point along the arm backbone is not important if the DTD process is faster than the hopping process. This situation is realized if the hopping time $\left(\tau_{\mathrm{w}}\right)$ is longer than the relaxation time of the backbone $\left(\tau_{\mathrm{b}}\right)$. For example, in the sliplink simulation by Shanbhag and Larson ${ }^{10}$ the hopping takes place on the full removal of sliplinks for a given arm (without reformation). They pointed out that in this case the resultant $\tau_{\mathrm{w}}$ is much longer than the end-to-end relaxation time of the arm $\left(\tau_{\mathrm{a}}\right)$ because the latter corresponds to the characteristic time for full renewal of sliplinks (including the reformed ones). Thus according to their model $\tau_{\mathrm{w}}$ may become larger than $\tau_{\mathrm{b}}$ if the backbone length is not sufficiently longer than the arm length. For the case of $\tau_{\mathrm{w}}>\tau_{\mathrm{b}}$, the available hierarchical models assuming the obligatory occurrence of curvilinear hopping could give a very low apparent mobility of the branch point $\left(p^{2} \ll 1\right)$ when applied to the data for those star polymers, because the apparent contribution of the branch point diffusion $\left(D_{\mathrm{b}}\right)$ to the main chain diffusion becomes relatively low.

Nevertheless, further analysis on the basis of those models requires a self-consistent DTD treatment (partial-DTD treatment), i.e., the coarse-graining of the length scale being consistent with the constraint release (CR) time scale. This treatment is possible if the detail of the CR process is experimentally known (e.g., for the case of symmetric star polymers ${ }^{16,17}$ ), but this is not the case for the asymmetric star polymers. Thus, at this moment, it is highly desired to examine the branch point motion without the coarse-graining, i.e., without starting from the DTD picture.

Thus, for the asymmetric star polymers, we conducted 3D multi-chain sliplink simulation (primitive chain network simulations formulated previously ${ }^{18,19}$ ) not based on the DTD picture to investigate the contribution of the branch point motion (both curvilinear hopping and the fluctuation) to the viscoelastic relaxation. It turned out that the fluctuation of the branch point is much more important compared to the curvilinear hopping when the lengths of the short and long backbone arms are not very different and the waiting time $\left(\tau_{\mathrm{w}}\right)$ for the branch point hopping (time for removal of all sliplinks on the short arm) is larger than the backbone relaxation time. Details of these results are presented in this paper.

\section{MODEL AND SIMULATIONS}

The simulation code utilized in this study has been proposed earlier (to consider a topological change around branch point). ${ }^{19}$ The polymer is represented by consecutive segments, sliplinks, and branch point. The segment molecular weight is assumed to be comparable to the entanglement molecular weight. The sliplink bundles segments in pair to form a network in 3D space. The polymer motion is described by Langevin-type equations for the spatial displacement of sliplinks, chain ends and branch points, and for a change of the monomer number in each segment due to the monomer transport along the chain backbone through the sliplink. The equations incorporate the chain elasticity, the chemical potential gradient, the friction against the medium, and the Brownian random force. For the three-arm asymmetric star polymers, the friction of the branch point is assumed to be $3 / 4$ of that of sliplink so as to make consistency with the number ( = 3) of segments converging to the branch point and the number for the sliplink (four segments from two chains).

In the simulation, the branch point motion and the chain sliding were allowed, and a topological change of the network was activated through creation and destruction of the sliplinks at the chain ends. A further topological change was activated around the branch point to realize the curvilinear hopping of the branch point along the backbone. ${ }^{19}$ The hopping was allowed when all sliplinks of an arm are lost simultaneously (full removal). Note that this hopping algorism is common to that proposed by Shanbhag and Larson ${ }^{10}$ and the resultant waiting time for the hopping $\left(\tau_{\mathrm{w}}\right)$ becomes much larger than the end-to-end relaxation time of the arm $\tau_{\mathrm{a}}$ (required for full renewal of all sliplinks). The curvilinear hopping of the branch point was achieved by a jump to the next segment across the neighboring sliplink, and thus the hopping distance was set equal to the undilated network mesh size. We assumed equal probability (1/3) for the choices among three events, the jumps toward two backbone directions and no jump. The last choice (no jump) was incorporated to represent an event that the retracted arm does not penetrate through the next sliplink but hooks another surrounding chain. The earlier study ${ }^{19}$ described the general procedure including convergence of more than two arms to the branch point. In some simulation runs, the hopping algorism was turned off to investigate the effect of the curvilinear hopping in the clearest way.

In the actual simulation runs, the average equilibrium length of the segment $a$ (the undilated network mesh size) was chosen as the unit length, $k T$ as the unit energy, $\tau_{0}$ $=\zeta a^{2} / 6 k T$ with $\zeta=$ friction coefficient of the sliplink as the unit time. For comparison with the experimental data, we utilized the unit of molecular weight $M_{0}$ (and the corresponding unit stress $G_{0}$ ) as a fitting parameter rather than the molecular weight calculated from the unit length $a$. The periodic boundary condition was employed with the simulation cell box of $8^{3}$ that contained $10 \times 8^{3}$ segments. Simulations under quiescent condition were performed for sufficient long time (at least 10 times longer than the terminal relaxation time), and the linear relaxation modulus $G(t)$ was obtained as the auto-correlation function of the microscopic shear stress. This auto-correlation function was calculated by the fast 




FIG. 1. Linear viscoelastic behavior of linear, symmetric star, and asymmetric star polymers from top to bottom. Solid and dashed curves show simulated $G^{\prime}$ and $G^{\prime \prime}$, respectively, and the symbols are taken from Ref. 20.

multi-tau correlator algorism, ${ }^{21}$ and the resulting $G(t)$ was converted into the complex modulus $G^{*}(\omega)$ with the REPTATE software. ${ }^{22}$

\section{RESULTS}

Figure 1 compares the storage and loss moduli, $G^{\prime}$ and $G^{\prime \prime}$, simulated for linear and symmetric/asymmetric star polymers with the experimental data reported by Lee et al. ${ }^{20}$ for high-M 1,4-polyisoprene (PI) dissolved in (blended with) a low-M, non-entangling PI with the high-M polymer volume fraction of $60 \%$. The molecular architecture and the sample code name are summarized in Table I. The simulation parameters to fit the data were $G_{0}=0.29 \mathrm{MPa}, M_{0}=6.5 \mathrm{kDa}$ and $\tau_{0}=3.1 \mathrm{msec}$. The relationship $M_{0}=\rho R T / G_{0}$ is satisfied for the simulation results, and the $M_{0}$ value is ca. $2 / 3$ of the value used in the tube theory, as noted in the earlier studies for linear polymers. ${ }^{23,24}$ As seen in Fig. 1, the simulation describes the data to an extent similar to that for the earlier theory presented in Ref. 20. However, it should be emphasized that our simulations included no additional parameter such as $p^{2}$ (utilized in the earlier study). The agreement seen in Fig. 1 is consistent with our earlier results ${ }^{17,25}$ describing the self-diffusion of star polymers with a parameter set common to linear polymers.

The agreement seen in Fig. 1 allows us to simulate the effects of the branch point motion (spatial fluctuation and the curvilinear hopping) on the viscoelastic relaxation. For the symmetric and asymmetric star polymers examined in Fig. 1, the modulus $G(t)$ simulated for the cases of both curvilinear hopping and fluctuation of the branch point, only the branch point fluctuation and no motion of the branch point are shown in Fig. 2 with the circles, dotted curves, and solid curves, respectively. For the symmetric star polymer (top panel), the
TABLE I. Architecture of the polymers examined in Figs. 1-4. $Z$ indicates average number of entanglement segments for the short arm $\left(Z_{\mathrm{a}}\right)$, the backbone $\operatorname{arm}\left(Z_{\mathrm{b}}\right)$ and the molecule as a whole $\left(Z_{\mathrm{t}}\right)$. Sample code corresponds to Ref. 20 .

\begin{tabular}{llccc}
\hline \hline Code & Architecture & $Z_{\mathrm{a}}$ & $Z_{\mathrm{b}}$ & $Z_{\mathrm{t}}$ \\
\hline N250K60 & Linear & $\ldots$ & 20 & 40 \\
S101K60 & Symmetric star & $\ldots$ & 16 & 48 \\
A2B73K60 & Asymmetric star & 5 & 11 & 26 \\
A2B182K60 & Asymmetric star & 5 & 28 & 61 \\
\hline \hline
\end{tabular}

branch point motion does not affect the relaxation. On the other hand, for the asymmetric stars (middle and bottom panels), the relaxation is decelerated in the case of absence of the branch point motion, as expected.

Table II summarizes the waiting time for hopping $\left(\tau_{\mathrm{w}}\right)$ and the end-to-end relaxation time for short $\left(\tau_{\mathrm{a}}\right)$ and backbone $\left(\tau_{\mathrm{b}}\right)$ arms for the asymmetric star polymers. $\tau_{\mathrm{w}}$ was evaluated from the simulation data for an accumulated number of the hopping events per molecule, $A_{\text {hop }}$. Figure 3 shows time development of $A_{\text {hop }}$ for the asymmetric star polymers examined in Figs. 1 and 2. Clearly, $A_{\text {hop }}$ is in proportion to $t$ at long times and the waiting time was evaluated as $\tau_{\mathrm{w}}=t / A_{\text {hop }}$ at long $t$. For A2B73K60 with short backbone arms, $\tau_{\mathrm{w}}>\tau_{\mathrm{b}}$ (cf. Table II) and thus the curvilinear hopping is too slow to affect the relaxation of the polymer. This result is consistent with the observation in the middle panel of Fig. 2 that $G(t)$ of A2B73K60 with and without the hopping mechanism (thick solid curve and dotted curve) coincide with each other. On the other hand, A2B182K60 with long backbone arms has $\tau_{\mathrm{w}}$ $\ll \tau_{\mathrm{b}}$ (cf. Table II) and thus the curvilinear diffusion reasonably dominates the relaxation. This behavior is reflected in the difference of $G(t)$ with and without the hopping mechanism seen in the bottom panel of Fig. 2. The relaxation times, $\tau_{\mathrm{a}}$ and $\tau_{\mathrm{w}}$, depend on the constraint release time being affected



FIG. 2. Simulated relaxation modulus of the branch polymers examined in Fig. 1. The circle, dotted curve, and thin solid curve, respectively, show the modulus for a case of both curvilinear hopping and fluctuation of the branch point, only the branch point fluctuation and no motion of the branch point. 
TABLE II. Characteristic times for the asymmetric star polymers in Figs. 1-4.

\begin{tabular}{lccc}
\hline \hline Code & $\tau_{\mathrm{a}}(\mathrm{sec})$ & $\tau_{\mathrm{w}}(\mathrm{sec})$ & $\tau_{\mathrm{b}}(\mathrm{sec})$ \\
\hline A2B73K60 & 0.94 & 3.0 & 1.4 \\
A2B182K60 & 1.2 & 4.4 & 63 \\
\hline \hline
\end{tabular}

by the backbone arm length, which results in the difference between A2B73K60 and A2B182K60.

One may argue that the curvilinear hopping of the branch point in our simulation algorithm is the hopping in the undilated (skinny) entanglement network and therefore the hopping may have still occurred in a dilated network (coarsegrained network in long time scales) even if this algorithm is turned off. To check this possibility, we compared the mean-square displacement (MSD) of the branch point and the squared mesh size $a^{\prime}(t)^{2}$ of the fully dilated network, the latter being defined in terms of the survival probability of the sliplinks, $\varphi(t)$. Since the squared end-to-end distance of a chain does not change on the dilation of network, we find

$$
Z a^{2}=Z \varphi(t) a^{\prime}(t)^{2} .
$$

Here $Z$ is the number of entanglement segment of the chain and $a$ is the undilated mesh size. From Eq. (1) we obtain the full-DTD expression (with the dilation exponent $=1$ ),

$$
a^{\prime}(t)^{2}=a^{2} / \varphi(t) .
$$

Figure 4 compares $a^{\prime}(t)^{2}$ (dash-dot curve) with MSD of the branch point (circle). For the linear polymer (top panel), MSD is shown for the monomer at the chain center. This MSD is larger than $a^{\prime}(t)^{2}$ in a range of $t$ up to the longest relaxation time, indicating that the chain diffuses through the dilated network via reptative motion. Indeed, the power-law exponent of MSD against $t$ is less than unity (and the center monomer exhibits non-Fickian behavior) in the range of $t$ where MSD $>a^{\prime}(t)^{2}$, which is consistent with the reptation model. ${ }^{2}$

Similar behavior is observed for the asymmetric star with long backbone (A2B182K60); cf. circle and thick dash-dot curve in the lowest panel of Fig. 4. This result confirms that

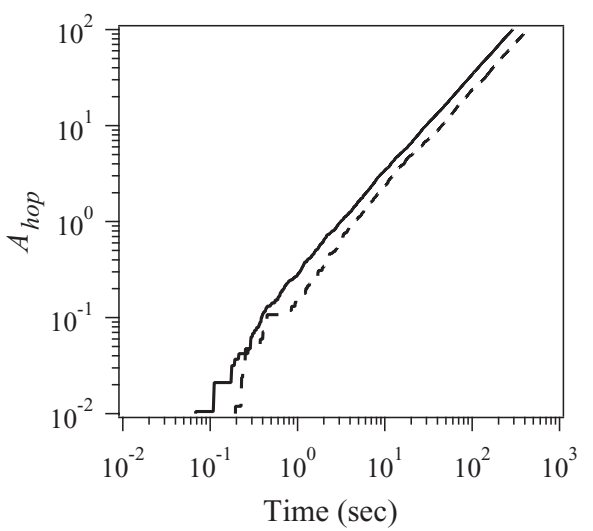

FIG. 3. Time evolution of the accumulated number of the curvilinear hopping per molecule obtained from the simulation data. Solid and broken lines show $\mathrm{A} 2 \mathrm{~B} 73 \mathrm{~K} 60$ and $\mathrm{A} 3 \mathrm{~B} 182 \mathrm{~K} 60$, respectively.

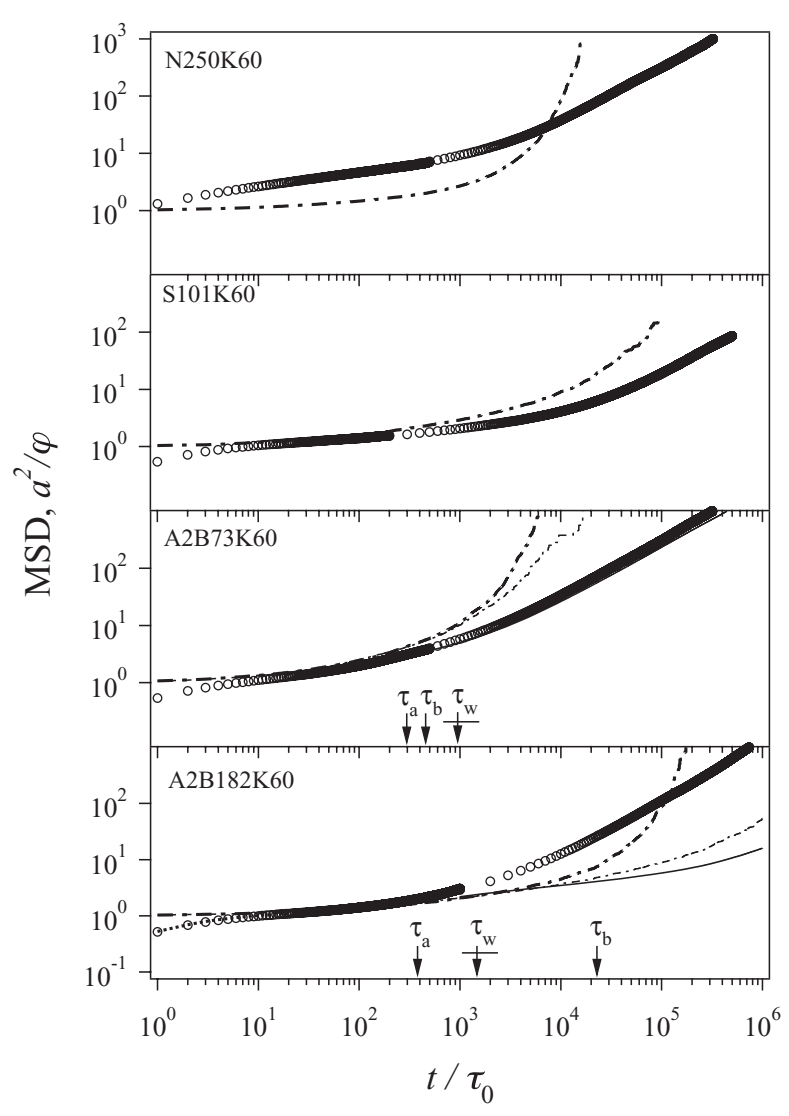

FIG. 4. Mean square displacement of the branch point (circle) and squared mesh size of fully dilated network $a^{\prime}(t)^{2}=a^{2} / \varphi(t)$ (thick dash-dot curve) for the polymers examined in Fig. 1. For branched polymers, MSD and $a^{\prime}(t)^{2}$ without the curvilinear hopping mechanism are shown by thin solid and dashdot curves.

the curvilinear hopping of the branch point affects the viscoelastic relaxation, as noted for $G(t)$ in the bottom panel of Fig. 2. Indeed, for A2B182K60, the MSD exceeds $a^{\prime}(t)^{2}$ at $t$ close to the waiting time for hopping $\tau_{\mathrm{w}}$, indicating that the branch point is essentially trapped in the undilated mesh before the branch point hops. On the contrary, when the hopping algorism is turned off, the MSD is always smaller than $a^{\prime}(t)^{2}$ (cf. the thin solid and dash-dot curves) and thus the curvilinear hopping does not occur even through the dilated network.

For the symmetric star (S101K60) and the asymmetric star with shorter backbone (A2B73K60) examined in the second and third panels of Fig. 4, MSD is always smaller than $a^{\prime}(t)^{2}$. This result indicates that the branch point motion is trapped in the dilated entanglement mesh. This feature is noted for both cases of the presence and absence of the hopping mechanism, which is in harmony with the observation for $G(t)$. Indeed, the results of simulations with and without the hopping algorism agreed with each other for the symmetric S101K60. For the asymmetric A2B73K60, the effect of the hopping is observed only at $t>\tau_{\mathrm{b}}$, where the relaxation of $G(t)$ almost completes.

We tested validity of the above argument for two groups of asymmetric star polymers having (i) various lengths of the short arm $Z_{\mathrm{a}}$ and a fixed length of the backbone arm $\left(Z_{\mathrm{b}}\right.$ 




FIG. 5. Relaxation modulus for a series of asymmetric star polymers with various lengths of short arm $\left(Z_{\mathrm{a}}\right)$ and a fixed length of backbone arm $\left(Z_{\mathrm{b}}\right.$ $=16$, and the backbone length is $2 Z_{\mathrm{b}}=32$ ). Circles are for simulations with the fluctuation and hopping mechanisms of branch point motion, thin dashed curves are only with the fluctuation mechanism, and thin solid curve is without any branch point motion.

$=16)$ and (ii) various $Z_{\mathrm{b}}$ and a fixed $Z_{\mathrm{a}}(=5)$. The simulated $G(t)$, MSD, and $a^{\prime}(t)^{2}$ are shown in Figs. 5 and 6 for the group (i) and in Figs. 7 and 8 for the group (ii). For the star polymer with the shortest arm examined in the group (i) $\left(Z_{\mathrm{a}}\right.$ $=2$; top panel of Fig. 5), the relaxation of $G(t)$ is insensitive to

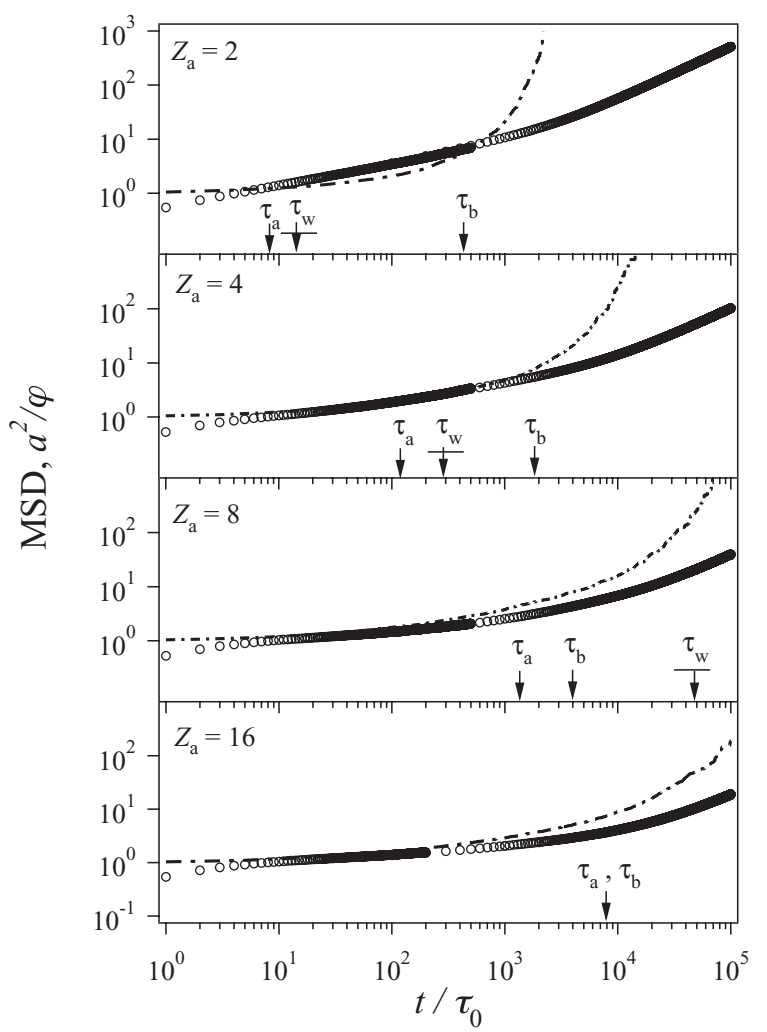

FIG. 6. Mean square displacement MSD of the branch point (circle) and squared mesh size of fully dilated network $a^{\prime}(t)^{2}=a^{2} / \varphi(t)$ (dash-dot curves) for the asymmetric star polymers examined in Fig. 5.

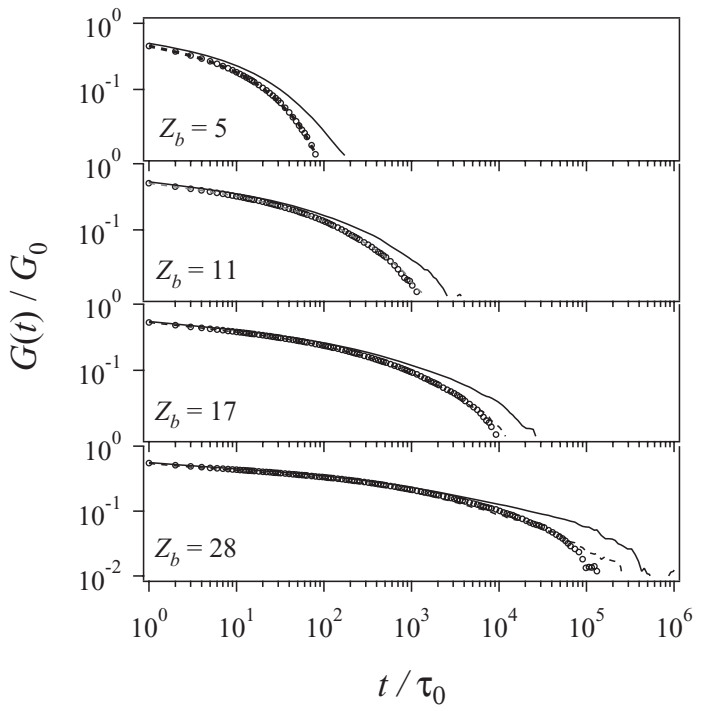

FIG. 7. Relaxation modulus for a series of asymmetric star polymers with various lengths of backbone $\operatorname{arm}\left(Z_{\mathrm{b}}\right)$ and a fixed length of short $\operatorname{arm}\left(Z_{\mathrm{a}}=5\right)$. Circles are for simulations with the fluctuation and hopping mechanisms of branch point motion, thin dashed curves are only with the fluctuation mechanism, and thin solid curve is without any branch point motion.

the fluctuation mechanism (cf. thin solid and dashed curves) but is significantly accelerated when the hopping mechanism is incorporated (circle). Indeed, $\tau_{\mathrm{w}} \ll \tau_{\mathrm{b}}$ for this case (cf. top panel of Fig. 6), confirming that the curvilinear hopping is the essential mechanism of terminal relaxation for this case.

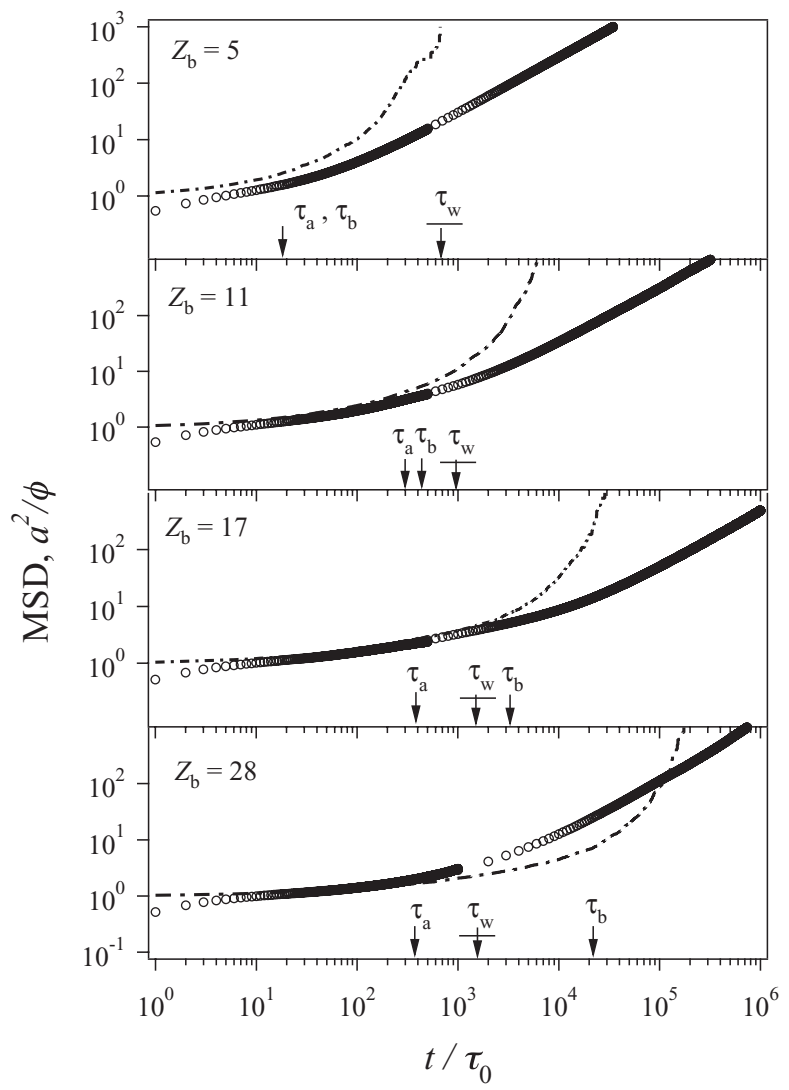

FIG. 8. Mean square displacement MSD of the branch point (circle) and squared mesh size of fully dilated network $a^{\prime}(t)^{2}=a^{2} / \varphi(t)$ (dash-dot curves) for the asymmetric star polymers examined in Fig. 7. 
For the star polymers with $Z_{\mathrm{a}}=4$ and 8 , the relaxation is rather insensitive to the hopping but considerably affected by the branch point fluctuation, as noted in the second and third panels of Fig. 5. As noted in Fig. 6, $\tau_{\mathrm{w}}$ is smaller than $\tau_{\mathrm{b}}$ but the difference between these times is rather small for $Z_{\mathrm{a}}=4$, while $\tau_{\mathrm{w}}>\tau_{\mathrm{b}}$ for $Z_{\mathrm{a}}=8$. For this reason, the curvilinear hopping of the branch point is not effectively contributing to the relaxation, as seen in Fig. 5. Finally, for $Z_{\mathrm{a}}=16$ ( $=Z_{\mathrm{b}}$; symmetric star $) \tau_{\mathrm{w}}\left(\sim 3.6 \times 10^{8}\right.$; out of the plot scale $)$ is orders of magnitude longer than $\tau_{\mathrm{b}}$ so that neither the fluctuation nor hopping of the branch point affects the relaxation, which naturally results in the behavior of $G(t)$ seen in the bottom panel of Fig. 5.

For the group (ii) examined in Fig. 7, the curvilinear hopping of the branch point contributes to the relaxation only for the case of the largest $Z_{\mathrm{b}}(=28)$. For this case, the reptative motion is indeed confirmed for MSD (see the lowest panel of Fig. 8). For the smaller $Z_{\mathrm{b}}$ values, $\tau_{\mathrm{b}}$ is not sufficiently larger than $\tau_{\mathrm{w}}$ (cf. Fig. 8), so that the hopping hardly contributes to $G(t)$ and the MSD is always smaller than $a^{\prime}(t)^{2}$ of the fully dilated entanglement mesh. Thus, the results seen in Figs. 5-8 suggest that the effect of the branch point hopping on the relaxation changes with the $\tau_{\mathrm{b}} / \tau_{\mathrm{w}}$ ratio.

\section{DISCUSSION}

The parameter $p^{2}$ is incorporated in the hierarchical tube model but not in our simulation. Nevertheless, the $p^{2}$ value can be still estimated from the simulation results. Within the tube model, the squared hopping distance is expressed as $\left(p a_{\mathrm{eff}}\right)^{2}$ with $a_{\mathrm{eff}}$ being the effectively dilated tube diameter. This $a_{\text {eff }}$ is equivalent to $a^{\prime}(t)$ specified by Eq. (2). Since the hopping occurs at $t=\tau_{\mathrm{w}}$, we can estimate $p^{2}$ in our simulation from the value of $1 / \varphi$ at $t=\tau_{\mathrm{w}}$. The $p^{2}$ thus estimated was around 1 to $1 / 2$ for the polymers relaxing under strong influence of the hopping mechanism (i.e., for A2B182K60 and the polymer with $Z_{\mathrm{a}}=2$ examined in Figs. 5 and 6). Although this $p^{2}$ value is much larger than the values reported on the basis of the tube model, it is self-consistent within the simulation. On the other hand, we found much smaller $p^{2}$ for the polymers not affected by the hopping but by the branch point fluctuation mechanism; for example, $p^{2} \cong 1 / 10$ for A2B73K60. As judged from this result, the very slow branch point motion reported in literature could be partly due to the application of the hierarchical tube model to the polymers that relax under strong influence of the branch point fluctuation but not of the curvilinear hopping.

\section{CONCLUSION}

The primitive chain network simulations were conducted for asymmetric star polymers to examine the contributions of the spatial fluctuation and curvilinear hopping of the branch point to the relaxation. The viscoelastic data of linear, symmetric star and asymmetric star polymers were quantitatively reproduced by the simulations that allowed both fluctuation and hopping of the branch point and utilized a common set of parameters. The simulations without the branch point motion predicted slower relaxation for asymmet- ric star polymers, demonstrating the significance of the branch point motion on the relaxation. Further tests were made for asymmetric star polymers with and without the curvilinear hopping of the branch point. For the polymers having the short and backbone arms of comparable lengths, the hopping was not effective but the branch point fluctuation within the dilated entanglement mesh significantly contributed to the viscoelastic relaxation. The importance of the curvilinear hopping was found only for a case that the backbone relaxation time is larger than the waiting time of hopping. The hopping distance parameter $p^{2}$ utilized in the tube model was estimated from the simulation results. The estimated $p^{2}$ values suggested that the very slow branch point motion reported in literature could be partly due to the application of the hierarchical tube model to the polymers relaxing under strong influence of the branch point fluctuation but not of the curvilinear hopping.

It should be emphasized that the observed significance of the branch point fluctuation arised from our rule for the curvilinear point hopping. Specifically, we assumed that the hopping occurs on full (simultaneous) removal of sliplinks for an arm. In this case the hopping time becomes much larger than the end-to-end relaxation time required for full renewal of the sliplinks. ${ }^{10}$ The results certainly vary if we assume another rule for the hopping. Thus, the model needs to be tested for other sets of experimental data so as to settle this problem.

It should be also noted that multi-branch polymers such as $\mathrm{H}$, pom-pom and comb polymers unequivocally exhibit the branch point hopping in the terminal relaxation regime because the entanglement between backbones cannot be released until the branch point hops. ${ }^{19}$ In this case, the effect of branch point fluctuation would be less significant compared to the effect for asymmetric stars. The simulation for those multi-branched polymers, including a test of the validity of the hopping rule, is now being conducted. The results will be published in our future paper.

${ }^{1}$ M. Doi and N. Y. Kuzuu, J. Polym. Sci. Part C: Polym. Lett. 18 (12), 775(1980).

${ }^{2}$ M. Doi and S. F. Edwards, The Theory of Polymer Dynamics (Clarendon, Oxford; Oxford University Press, New York, 1986).

${ }^{3}$ L. J. Fetters, A. D. Kiss, D. S. Pearson, G. F. Quack, and F. J. Vitus, Macromolecules 26 (4), 647(1993).

${ }^{4}$ D. S. Pearson and E. Helfand, Macromolecules 17 (4), 888 (1984).

${ }^{5}$ R. C. Ball and T. C. B. McLeish, Macromolecules 22 (4), 1911 (1989).

${ }^{6}$ S. T. Milner and T. C. B. McLeish, Macromolecules 31 (21), 7479 (1998).

${ }^{7}$ T. C. B. McLeish, J. Allgaier, D. K. Bick, G. Bishko, P. Biswas, R. Blackwell, B. Blottiere, N. Clarke, B. Gibbs, D. J. Groves, A. Hakiki, R. K. Heenan, J. M. Johnson, R. Kant, D. J. Read, and R. N. Young, Macromolecules 32 (20), 6734 (1999).

${ }^{8}$ R. G. Larson, Macromolecules 34 (13), 4556 (2001).

${ }^{9}$ A. L. Frischknecht, S. T. Milner, A. Pryke, R. N. Young, R. Hawkins, and T. C. B. McLeish, Macromolecules 35 (12), 4801 (2002).

${ }^{10}$ S. Shanbhag and R. G. Larson, Macromolecules 37 (21), 8160 (2004).

${ }^{11}$ J. H. Lee, L. J. Fetters, and L. A. Archer, Macromolecules 38 (10), 4484 (2005).

${ }^{12}$ S. J. Park, S. Shanbhag, and R. G. Larson, Rheol. Acta 44 (3), 319 (2005).

${ }^{13}$ E. van Ruymbeke, C. Bailly, R. Keunings, and D. Vlassopoulos, Macromolecules 39 (18), 6248 (2006).

${ }^{14}$ Q. Zhou and R. G. Larson, Macromolecules 40 (9), 3443 (2007).

${ }^{15}$ M. Zamponi, W. Pyckhout-Hintzen, A. Wischnewski, M. Monkenbusch, L. Willner, G. Kali, and D. Richter, Macromolecules 43 (1), 518 (2010). 
${ }^{16} \mathrm{H}$. Watanabe, T. Sawada, and Y. Matsumiya, Macromolecules 39(7), 2553 (2006).

${ }^{17}$ X. Qiao, T. Sawada, Y. Matsumiya, and H. Watanabe, Macromolecules 39(21), 7333 (2006).

${ }^{18}$ Y. Masubuchi, J. I. Takimoto, K. Koyama, G. Ianniruberto, G. Marrucci, and F. Greco, J. Chem. Phys. 115 (9), 4387 (2001).

${ }^{19}$ Y. Masubuchi, G. Ianniruberto, F. Greco, and G. Marrucci, Rheol. Acta 46 (2), 297 (2006).

${ }^{20}$ J. H. Lee, J. M. Goldberg, L. J. Fetters, and L. A. Archer, Macromolecules 39 (19), 6677 (2006).
${ }^{21}$ D. Magatti and F. Ferri, Appl. Opt. 40 (24), 4011 (2001).

${ }^{22}$ See http://reptate.com by J. Ramirez and A. Likhtman (2009) for information about the REPTATE software.

${ }^{23}$ Y. Masubuchi, G. Lanniruberto, F. Greco, and G. Marrucci, J. NonNewtonian Fluid Mech. 149 (1-3), 87 (2008).

${ }^{24}$ Y. Masubuchi, K. Furuichi, K. Horio, T. Uneyama, H. Watanabe, G. Ianniruberto, F. Greco, and G. Marrucci, J. Chem. Phys. 131 (11), 114906 (2009).

${ }^{25}$ Y. Masubuchi, G. Ianniruberto, F. Greco, and G. Marrucci, Modell. Simul. Mater. Sci. Eng. 12 (3), S91-S100 (2004). 